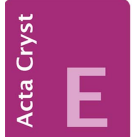
COMMUNICATIONS

ISSN 2056-9890

\section{Crystal structure of a methimazole-based ionic liquid}

Jamie C. Gaitor, ${ }^{a}$ Manuel Sanchez Zayas, ${ }^{\text {a }}$ Darrel J. Myrthil, ${ }^{a}$ Frankie White, ${ }^{\mathrm{b}}$ Jeffrey M. Hendrich, ${ }^{\mathrm{b}}$ Richard E. Sykora, ${ }^{b}$ Richard A. O'Brien, ${ }^{b}$ John T. Reilly a and Arsalan Mirjafari $^{\mathrm{a} *}$

a Department of Chemistry and Physics, Florida Gulf Coast University, Fort Myers, FL 33965, USA, and 'buniversity of South Alabama, Department of Chemistry, Mobile, AL 36688, USA. *Correspondence e-mail: amirjafari@fgcu.edu

Received 13 November 2015; accepted 19 November 2015

Edited by P. C. Healy, Griffith University, Australia

The structure of 1-methyl-2-(prop-2-en-1-ylsulfanyl)- $1 H$ imidazol-3-ium bromide, $\mathrm{C}_{7} \mathrm{H}_{11} \mathrm{~N}_{2} \mathrm{~S}^{+} \cdot \mathrm{Br}^{-}$, has monoclinic $\left(P 2_{1} / c\right)$ symmetry. In the crystal, the components are linked by $\mathrm{N}-\mathrm{H} \cdots \mathrm{Br}$ and $\mathrm{C}-\mathrm{H} \cdots \mathrm{Br}$ hydrogen bonds. The crystal structure of the title compound undeniably proves that methimazole reacts through the thione tautomer, rather than the thiol tautomer in this system.

Keywords: crystal structure; ionic liquids; methimazole; S-allylation; nitrogen heterocycle.

CCDC reference: 1437865

\section{Related literature}

For the biological activity of methimazole, see: Rong et al. (2013). For its use as a ligand, see: Crossley et al. (2006). For a discussion of methimazole-based ionic liquids, see: Siriwardana et al. (2008). For reaction chemistry of methimazole, see: Roy \& Mugesh (2005).

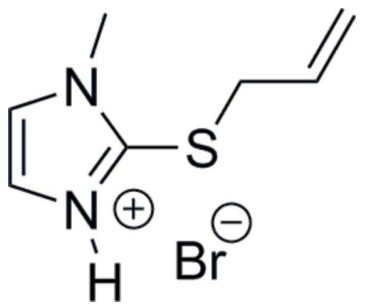

\section{Experimental}

2.1. Crystal data

$\mathrm{C}_{7} \mathrm{H}_{11} \mathrm{~N}_{2} \mathrm{~S}^{+} \cdot \mathrm{Br}^{-}$

$M_{r}=235.15$

Monoclinic, $P 2_{1} / c$

$a=10.8692(7) \AA$

$b=7.4103(5) \AA$

$c=12.8551(9) \AA$

$\beta=104.006(7)^{\circ}$

$V=1004.62(11) \AA^{3}$

$Z=4$

Mo $K \alpha$ radiation

$\mu=4.24 \mathrm{~mm}^{-1}$

$T=180 \mathrm{~K}$

$0.6 \times 0.32 \times 0.25 \mathrm{~mm}$

\subsection{Data collection}

Agilent Xcalibur, Eos diffractometer

Absorption correction: multi-scan (CrysAlis PRO; Agilent, 2014) $T_{\min }=0.321, T_{\max }=1.000$

7388 measured reflections 1829 independent reflections 1558 reflections with $I>2 \sigma(I)$ $R_{\text {int }}=0.042$

\subsection{Refinement}

$R\left[F^{2}>2 \sigma\left(F^{2}\right)\right]=0.030$

$w R\left(F^{2}\right)=0.065$

$S=1.03$

1829 reflections

105 parameters

1 restraint

$\mathrm{H}$ atoms treated by a mixture of independent and constrained refinement

$\Delta \rho_{\max }=0.34$ e $\AA^{-3}$

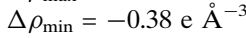

Table 1

Hydrogen-bond geometry $\left(\AA{ }^{\circ}\right)$.

\begin{tabular}{|c|c|c|c|c|}
\hline$D-\mathrm{H} \cdots A$ & $D-\mathrm{H}$ & $\mathrm{H} \cdots A$ & $D \cdots A$ & $D-\mathrm{H} \cdots A$ \\
\hline $\mathrm{N} 2-\mathrm{H} 2 \cdots \mathrm{Br} 1^{\mathrm{i}}$ & $0.84(3)$ & $2.46(3)$ & $3.246(2)$ & $158(3)$ \\
\hline $\mathrm{C} 2-\mathrm{H} 2 A \cdots \mathrm{Br} 1^{\mathrm{ii}}$ & 0.93 & 2.84 & $3.723(4)$ & 159 \\
\hline $\mathrm{C} 3-\mathrm{H} 3 \cdots \mathrm{Br} 1^{\mathrm{iii}}$ & 0.93 & 2.91 & $3.757(3)$ & 152 \\
\hline $\mathrm{C} 4-\mathrm{H} 4 B \cdots \mathrm{Br} 1$ & 0.96 & 2.87 & $3.737(3)$ & 151 \\
\hline $\mathrm{C} 5-\mathrm{H} 5 B \cdots \mathrm{Br} 1^{\mathrm{iv}}$ & 0.97 & 2.89 & $3.814(3)$ & 161 \\
\hline
\end{tabular}

Data collection: CrysAlis PRO (Agilent, 2014); cell refinement: CrysAlis PRO; data reduction: CrysAlis PRO; program(s) used to solve structure: SHELXS97 (Sheldrick, 2008); program(s) used to refine structure: SHELXL97 (Sheldrick, 2008); molecular graphics: OLEX2 (Dolomanov et al., 2009); software used to prepare material for publication: OLEX2 and publCIF (Westrip, 2010).

\title{
Acknowledgements
}

The authors thank the Alice and Karl Sheffield Scholarship and the Department of Chemistry and Physics of FGCU for funding this project. They also acknowledge the National Science Foundation for their generous support (NSFCAREER grant to RES, CHE-0846680).

Supporting information for this paper is available from the IUCr electronic archives (Reference: HG5463). 


\section{References}

Agilent (2014). CrysAlis PRO. Agilent Technologies, Yarnton, England.

Crossley, I. R., Hill, A. F., Humphrey, E. R. \& Smith, M. K. (2006). Organometallics, 25, 2242-2247.

Dolomanov, O. V., Bourhis, L. J., Gildea, R. J., Howard, J. A. K. \& Puschmann, H. (2009). J. Appl. Cryst. 42, 339-341.
Rong, Y., Al-Harbi, A., Kriegel, B. \& Parkin, G. (2013). Inorg. Chem. 52, 7172 7182.

Roy, G. \& Mugesh, G. (2005). J. Am. Chem. Soc. 127, 15207-15217.

Sheldrick, G. M. (2008). Acta Cryst. A64, 112-122.

Siriwardana, A. I., Crossley, I. R., Torriero, A. J., Burgar, I. M., Dunlop, N. F., Bond, A. M., Deacon, G. B. \& MacFarlane, D. R. (2008). J. Org. Chem. 73, 4676-4679.

Westrip, S. P. (2010). J. Appl. Cryst. 43, 920-925. 


\title{
supporting information
}

Acta Cryst. (2015). E71, o1008-o1009 [https://doi.org/10.1107/S2056989015022136]

\section{Crystal structure of a methimazole-based ionic liquid}

\author{
Jamie C. Gaitor, Manuel Sanchez Zayas, Darrel J. Myrthil, Frankie White, Jeffrey M. Hendrich, \\ Richard E. Sykora, Richard A. O'Brien, John T. Reilly and Arsalan Mirjafari
}

\section{S1. Comment}

2-Mercapto-1-methylimidazole or methimazole 1 belongs to a class of five-membered heterocyclic nitrogen compounds, which possess various biological activities (e.g. it is a widely used anti-thyroid drug under the name Tapazole), see: Rong et al. (2013). Additionally, it has found use as a multidentate ligand in the fields of inorganic and organometallic chemistry, in which the sulfur atom can serve as a soft donor towards a wide variety of transition metals, see: Crossley et al. (2006). The alkylation of methimazole with alkyl halides (e.g. iodoethane and chlorobutane) lead to the formation of methimazole-based ionic liquids in high yields, see Siriwardana et al. (2008). To date, no methimazole-based ionic liquids have been structurally characterized by X-ray diffraction.

Methimazole exists in two tautomeric forms, equilibrating between the 2-thiol 1a and 2-thione 1b, and both N-alkylation and S-alkylation reactions are possible, depending upon the reaction conditions and types of substrates employed, see Roy \& Mugesh (2005). They reported that only S-alkylated methimazoles were formed. The product structures were established by NMR spectroscopy, which is elusive in terms of proving the exclusive formation of S-alkylated products over N-alkylated products. Herein, we report the crystal structure of S-allylated methimazolium bromide 2, which was prepared in quantitative yield $(96 \%)$ via the reaction of methimazole with allyl bromide in refluxing acetonitrile (Scheme $\mathrm{S} 1)$. The crystal structure of $\mathbf{2}$ undeniably proves that methimazole reacts through the 2-thione tautomer $\mathbf{1 b}$.

\section{S2. Synthesis and crystallization}

2-Mercapto-1-methylimidazole $(0.57 \mathrm{~g}, 5 \mathrm{mmol})$ and allyl bromide $(0.85 \mathrm{~g}, 7 \mathrm{mmol})$ were dissolved in acetonitrile $(5.0$ $\mathrm{mL}$ ) and the mixture refluxed for 48 hours. The solvent and excess allyl bromide were removed under vacuum to afford an off-white solid. The solid was washed with toluene $(3 \times 10 \mathrm{~mL})$ and then recrystallized in acetonitrile to yield pure product 2 as an off-white solid in $96 \%$ isolated yield.

\section{S3. Refinement}

Crystal data, data collection and structure refinement details are summarized in Table 1. The H-atom (H2) located on N2 was allowed to freely refine (isotropically). The remaining $\mathrm{H}$-atoms were placed in calculated positions and allowed to ride during subsequent refinement, with $U_{\text {iso }}(\mathrm{H})=1.5 U_{\mathrm{eq}}(\mathrm{C})$ and $\mathrm{C}-\mathrm{H}$ distances of $0.96 \AA$ for methyl hydrogens, with $U_{\text {iso }}(\mathrm{H})=1.2 U_{\text {eq }}(\mathrm{C})$ and $\mathrm{C}-\mathrm{H}$ distances of $0.97 \AA$ for the secondary hydrogens, and with $U_{\text {iso }}(\mathrm{H})=1.2 U_{\text {eq }}(\mathrm{C})$ and $\mathrm{C}-\mathrm{H}$ distances of $0.93 \AA$ for all remaining hydrogen atoms. 


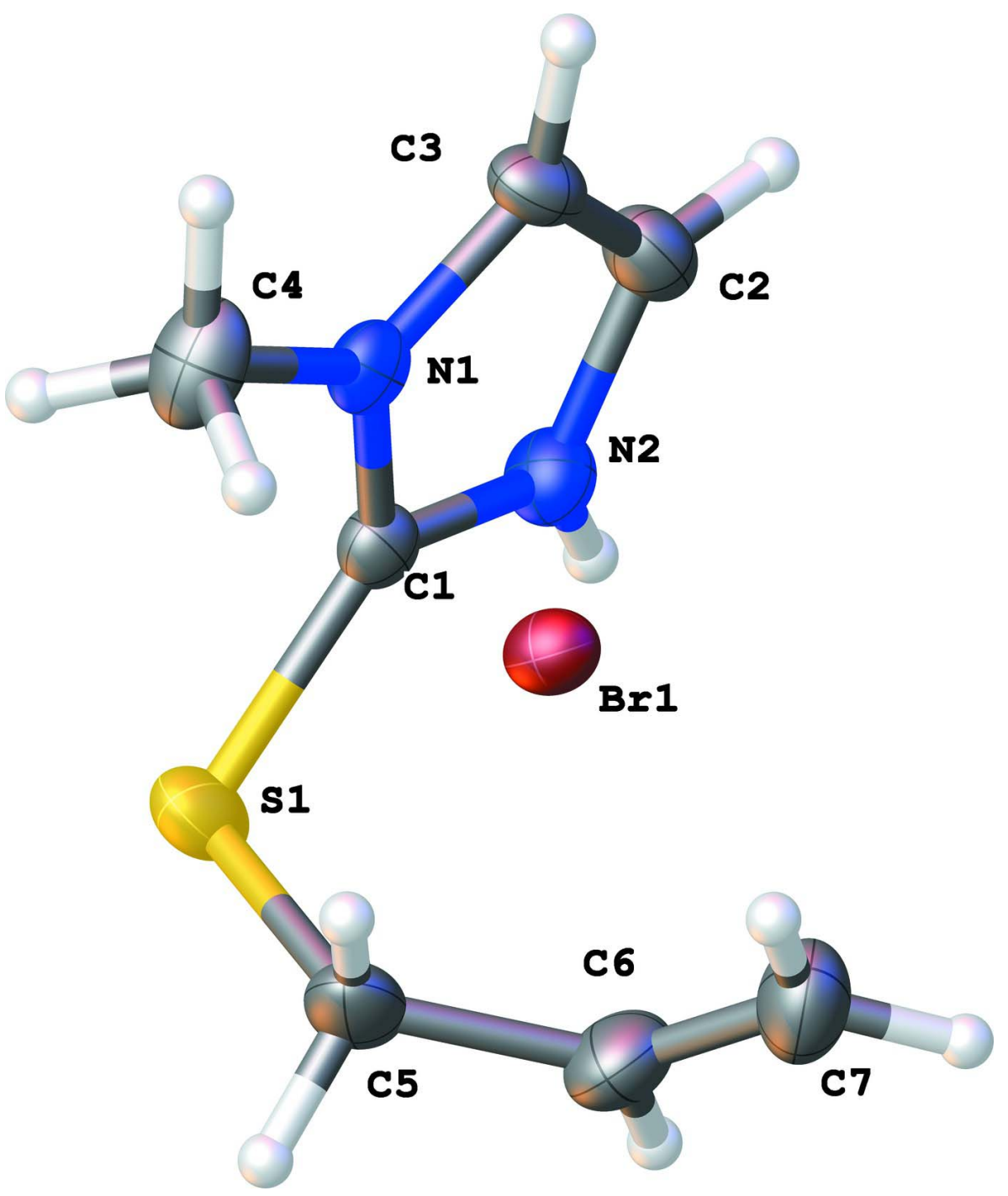

Figure 1

A thermal ellipsoid diagram of the structure of the title compound.<smiles>CCCSc1nccn1C</smiles>

1a<smiles>Cn1cc[nH]c1=S</smiles>

$1 b$

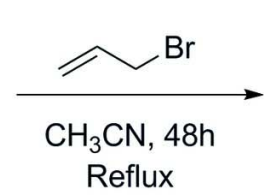

Reflux<smiles></smiles>

Figure 2

Reaction scheme.

1-Methyl-2-(prop-2-en-1-ylsulfanyl)-1 H-imidazol-3-ium bromide

Crystal data

$\mathrm{C}_{7} \mathrm{H}_{11} \mathrm{~N}_{2} \mathrm{~S}^{+} \cdot \mathrm{Br}^{-}$

$M_{r}=235.15$

Monoclinic, $P 2{ }_{1} / c$

$$
\begin{aligned}
& a=10.8692(7) \AA \\
& b=7.4103(5) \AA \\
& c=12.8551(9) \AA
\end{aligned}
$$


$\beta=104.006(7)^{\circ}$

$V=1004.62(11) \AA^{3}$

$Z=4$

$F(000)=472$

$D_{\mathrm{x}}=1.555 \mathrm{Mg} \mathrm{m}^{-3}$

Mo $K \alpha$ radiation, $\lambda=0.71073 \AA$

Data collection

Agilent Xcalibur, Eos

diffractometer

Radiation source: Enhance (Mo) X-ray Source

Graphite monochromator

Detector resolution: 16.0514 pixels $\mathrm{mm}^{-1}$

$\omega$ scans

Absorption correction: multi-scan

(CrysAlis PRO; Agilent, 2014)

$T_{\min }=0.321, T_{\max }=1.000$

Refinement

Refinement on $F^{2}$

Least-squares matrix: full

$R\left[F^{2}>2 \sigma\left(F^{2}\right)\right]=0.030$

$w R\left(F^{2}\right)=0.065$

$S=1.03$

1829 reflections

105 parameters

1 restraint

Primary atom site location: structure-invariant direct methods
Cell parameters from 2203 reflections

$\theta=3.9-27.0^{\circ}$

$\mu=4.24 \mathrm{~mm}^{-1}$

$T=180 \mathrm{~K}$

Prism, colourless

$0.6 \times 0.32 \times 0.25 \mathrm{~mm}$

7388 measured reflections

1829 independent reflections

1558 reflections with $I>2 \sigma(I)$

$R_{\text {int }}=0.042$

$\theta_{\text {max }}=25.3^{\circ}, \theta_{\text {min }}=3.2^{\circ}$

$h=-13 \rightarrow 13$

$k=-8 \rightarrow 8$

$l=-15 \rightarrow 15$

Secondary atom site location: difference Fourier map

Hydrogen site location: inferred from neighbouring sites

$\mathrm{H}$ atoms treated by a mixture of independent and constrained refinement

$w=1 /\left[\sigma^{2}\left(F_{\mathrm{o}}^{2}\right)+(0.027 P)^{2}\right]$

where $P=\left(F_{\mathrm{o}}^{2}+2 F_{\mathrm{c}}^{2}\right) / 3$

$(\Delta / \sigma)_{\max }=0.001$

$\Delta \rho_{\max }=0.34$ e $\AA^{-3}$

$\Delta \rho_{\min }=-0.38$ e $\AA^{-3}$

Special details

Experimental. CrysAlis Pro (Agilent, 2014) Empirical absorption correction using spherical harmonics, implemented in SCALE3 ABSPACK scaling algorithm.

Geometry. All e.s.d.'s (except the e.s.d. in the dihedral angle between two 1.s. planes) are estimated using the full covariance matrix. The cell e.s.d.'s are taken into account individually in the estimation of e.s.d.'s in distances, angles and torsion angles; correlations between e.s.d.'s in cell parameters are only used when they are defined by crystal symmetry. An approximate (isotropic) treatment of cell e.s.d.'s is used for estimating e.s.d.'s involving 1.s. planes.

Refinement. Refinement of $F^{2}$ against ALL reflections. The weighted $R$-factor $w R$ and goodness of fit $S$ are based on $F^{2}$, conventional $R$-factors $R$ are based on $F$, with $F$ set to zero for negative $F^{2}$. The threshold expression of $F^{2}>2 \sigma\left(F^{2}\right)$ is used only for calculating $R$-factors(gt) $e t c$. and is not relevant to the choice of reflections for refinement. $R$-factors based on $F^{2}$ are statistically about twice as large as those based on $F$, and $R$ - factors based on ALL data will be even larger.

Fractional atomic coordinates and isotropic or equivalent isotropic displacement parameters $\left(\hat{A}^{2}\right)$

\begin{tabular}{lllll}
\hline & $x$ & $y$ & $z$ & $U_{\text {iso }}^{*} / U_{\text {eq }}$ \\
\hline Br1 & $0.70880(3)$ & $0.66161(4)$ & $0.53045(2)$ & $0.02621(12)$ \\
$\mathrm{N} 2$ & $0.6562(2)$ & $0.0414(3)$ & $0.2392(2)$ & $0.0273(6)$ \\
$\mathrm{N} 1$ & $0.6302(2)$ & $0.1226(3)$ & $0.39296(18)$ & $0.0233(6)$ \\
$\mathrm{C} 2$ & $0.5297(3)$ & $0.0739(4)$ & $0.2258(3)$ & $0.0316(8)$ \\
$\mathrm{H} 2 \mathrm{~A}$ & 0.4669 & 0.0623 & 0.1625 & $0.038^{*}$ \\
$\mathrm{C} 3$ & $0.5134(3)$ & $0.1262(4)$ & $0.3221(3)$ & $0.0285(7)$ \\
$\mathrm{H} 3$ & 0.4371 & 0.1587 & 0.3376 & $0.034^{*}$
\end{tabular}




$\begin{array}{lllll}\text { C1 } & 0.7171(3) & 0.0704(4) & 0.3410(2) & 0.0234(7) \\ \text { S1 } & 0.87834(8) & 0.04183(11) & 0.39532(7) & 0.0373(2) \\ \text { C4 } & 0.6547(4) & 0.1637(4) & 0.5076(2) & 0.0370(9) \\ \text { H4A } & 0.5756 & 0.1749 & 0.5278 & 0.055^{*} \\ \text { H4B } & 0.7009 & 0.2750 & 0.5221 & 0.055^{*} \\ \text { H4C } & 0.7037 & 0.0681 & 0.5481 & 0.055^{*} \\ \text { C6 } & 0.9092(3) & 0.3747(4) & 0.3027(3) & 0.0410(9) \\ \text { H6 } & 0.9450 & 0.3274 & 0.2498 & 0.049^{*} \\ \text { C5 } & 0.9322(3) & 0.2790(4) & 0.4066(3) & 0.0405(9) \\ \text { H5A } & 0.8889 & 0.3429 & 0.4532 & 0.049^{*} \\ \text { H5B } & 1.0223 & 0.2818 & 0.4401 & 0.049^{*} \\ \text { C7 } & 0.8417(3) & 0.5215(5) & 0.2809(3) & 0.0436(9) \\ \text { H7A } & 0.8047 & 0.5719 & 0.3323 & 0.052^{*} \\ \text { H7B } & 0.8305 & 0.5758 & 0.2141 & 0.052^{*} \\ \text { H2 } & 0.689(3) & 0.007(4) & 0.190(2) & 0.049(11)^{*}\end{array}$

Atomic displacement parameters $\left(\AA^{2}\right)$

\begin{tabular}{lllllll}
\hline & $U^{11}$ & $U^{22}$ & $U^{33}$ & $U^{12}$ & $U^{13}$ & $U^{23}$ \\
\hline Br1 & $0.02573(18)$ & $0.0284(2)$ & $0.02529(19)$ & $-0.00350(13)$ & $0.00772(13)$ & $-0.00185(13)$ \\
$\mathrm{N} 2$ & $0.0309(15)$ & $0.0295(16)$ & $0.0227(15)$ & $0.0016(12)$ & $0.0088(13)$ & $-0.0005(12)$ \\
$\mathrm{N} 1$ & $0.0304(15)$ & $0.0165(13)$ & $0.0238(14)$ & $-0.0003(11)$ & $0.0081(12)$ & $0.0002(10)$ \\
$\mathrm{C} 2$ & $0.0256(17)$ & $0.030(2)$ & $0.0354(19)$ & $-0.0008(14)$ & $-0.0003(15)$ & $0.0026(15)$ \\
$\mathrm{C} 3$ & $0.0190(16)$ & $0.0255(18)$ & $0.041(2)$ & $0.0028(13)$ & $0.0064(14)$ & $0.0023(15)$ \\
$\mathrm{C} 1$ & $0.0253(17)$ & $0.0180(17)$ & $0.0264(17)$ & $-0.0015(13)$ & $0.0057(14)$ & $0.0027(13)$ \\
$\mathrm{S} 1$ & $0.0229(4)$ & $0.0335(5)$ & $0.0518(6)$ & $0.0031(4)$ & $0.0019(4)$ & $0.0112(4)$ \\
$\mathrm{C} 4$ & $0.059(2)$ & $0.028(2)$ & $0.0254(18)$ & $-0.0016(16)$ & $0.0128(17)$ & $-0.0035(14)$ \\
$\mathrm{C} 6$ & $0.039(2)$ & $0.045(2)$ & $0.044(2)$ & $-0.0026(17)$ & $0.0186(18)$ & $0.0108(18)$ \\
$\mathrm{C} 5$ & $0.0249(18)$ & $0.040(2)$ & $0.051(2)$ & $-0.0105(15)$ & $-0.0023(16)$ & $0.0134(17)$ \\
$\mathrm{C} 7$ & $0.049(2)$ & $0.042(2)$ & $0.037(2)$ & $0.0000(18)$ & $0.0056(18)$ & $0.0126(17)$ \\
\hline
\end{tabular}

Geometric parameters $\left(\AA,{ }^{o}\right)$

\begin{tabular}{llll}
\hline $\mathrm{N} 2-\mathrm{C} 2$ & $1.366(4)$ & $\mathrm{C} 4-\mathrm{H} 4 \mathrm{~A}$ & 0.9600 \\
$\mathrm{~N} 2-\mathrm{C} 1$ & $1.334(4)$ & $\mathrm{C} 4-\mathrm{H} 4 \mathrm{~B}$ & 0.9600 \\
$\mathrm{~N} 2-\mathrm{H} 2$ & $0.836(17)$ & $\mathrm{C} 4-\mathrm{H} 4 \mathrm{C}$ & 0.9600 \\
$\mathrm{~N} 1-\mathrm{C} 3$ & $1.373(4)$ & $\mathrm{C} 6-\mathrm{H} 6$ & 0.9300 \\
$\mathrm{~N} 1-\mathrm{C} 1$ & $1.338(3)$ & $\mathrm{C} 6-\mathrm{C} 5$ & $1.480(4)$ \\
$\mathrm{N} 1-\mathrm{C} 4$ & $1.465(4)$ & $\mathrm{C} 6-\mathrm{C} 7$ & $1.304(4)$ \\
$\mathrm{C} 2-\mathrm{H} 2 \mathrm{~A}$ & 0.9300 & $\mathrm{C} 5-\mathrm{H} 5 \mathrm{~A}$ & 0.9700 \\
$\mathrm{C} 2-\mathrm{C} 3$ & $1.349(4)$ & $\mathrm{C} 5-\mathrm{H} 5 \mathrm{~B}$ & 0.9700 \\
$\mathrm{C} 3-\mathrm{H} 3$ & 0.9300 & $\mathrm{C} 7-\mathrm{H} 7 \mathrm{~A}$ & 0.9300 \\
$\mathrm{C} 1-\mathrm{S} 1$ & $1.736(3)$ & $\mathrm{C} 7-\mathrm{H} 7 \mathrm{~B}$ & \\
$\mathrm{~S} 1-\mathrm{C} 5$ & $1.847(3)$ & & 109.5 \\
& & & 109.5 \\
$\mathrm{C} 2-\mathrm{N} 2-\mathrm{H} 2$ & $124(2)$ & $\mathrm{N} 1-\mathrm{C} 4-\mathrm{H} 4 \mathrm{~B}$ & 109.5 \\
$\mathrm{C} 1-\mathrm{N} 2-\mathrm{C} 2$ & $109.9(3)$ & $\mathrm{N} 1-\mathrm{C} 4-\mathrm{H} 4 \mathrm{C}$ & \\
$\mathrm{C} 1-\mathrm{N} 2-\mathrm{H} 2$ & $126(2)$ & $\mathrm{H} 4 \mathrm{~A}-\mathrm{C} 4-\mathrm{H} 4 \mathrm{~B}$ &
\end{tabular}




$\begin{array}{llll}\mathrm{C} 3-\mathrm{N} 1-\mathrm{C} 4 & 125.3(3) & \mathrm{H} 4 \mathrm{~A}-\mathrm{C} 4-\mathrm{H} 4 \mathrm{C} & 109.5 \\ \mathrm{C} 1-\mathrm{N} 1-\mathrm{C} 3 & 109.0(2) & \mathrm{H} 4 \mathrm{~B}-\mathrm{C} 4-\mathrm{H} 4 \mathrm{C} & 109.5 \\ \mathrm{C} 1-\mathrm{N} 1-\mathrm{C} 4 & 125.7(3) & \mathrm{C} 5-\mathrm{C} 6-\mathrm{H} 6 & 118.1 \\ \mathrm{~N} 2-\mathrm{C} 2-\mathrm{H} 2 \mathrm{~A} & 126.7 & \mathrm{C} 7-\mathrm{C} 6-\mathrm{H} 6 & 118.1 \\ \mathrm{C} 3-\mathrm{C} 2-\mathrm{N} 2 & 106.7(3) & \mathrm{C} 7-\mathrm{C} 6-\mathrm{C} 5 & 123.8(3) \\ \mathrm{C} 3-\mathrm{C} 2-\mathrm{H} 2 \mathrm{~A} & 126.7 & \mathrm{~S} 1-\mathrm{C} 5-\mathrm{H} 5 \mathrm{~A} & 108.8 \\ \mathrm{~N} 1-\mathrm{C} 3-\mathrm{H} 3 & 126.3 & \mathrm{~S} 1-\mathrm{C} 5-\mathrm{H} 5 \mathrm{~B} & 108.8 \\ \mathrm{C} 2-\mathrm{C} 3-\mathrm{N} 1 & 107.3(3) & \mathrm{C} 6-\mathrm{C} 5-\mathrm{S} 1 & 113.8(2) \\ \mathrm{C} 2-\mathrm{C} 3-\mathrm{H} 3 & 126.3 & \mathrm{C} 6-\mathrm{C} 5-\mathrm{H} 5 \mathrm{~A} & 108.8 \\ \mathrm{~N} 2-\mathrm{C} 1-\mathrm{N} 1 & 107.1(3) & \mathrm{C} 6-\mathrm{C} 5-\mathrm{H} 5 \mathrm{~B} & 107.7 \\ \mathrm{~N} 2-\mathrm{C} 1-\mathrm{S} 1 & 126.0(2) & \mathrm{H} 5 \mathrm{~A}-\mathrm{C} 5-\mathrm{H} 5 \mathrm{~B} & 120.0 \\ \mathrm{~N} 1-\mathrm{C} 1-\mathrm{S} 1 & 126.9(2) & \mathrm{C} 6-\mathrm{C} 7-\mathrm{H} 7 \mathrm{~A} & 120.0 \\ \mathrm{C} 1-\mathrm{S} 1-\mathrm{C} 5 & 100.68(14) & \mathrm{C} 6-\mathrm{C} 7-\mathrm{H} 7 \mathrm{~B} & 120.0 \\ \mathrm{~N} 1-\mathrm{C} 4-\mathrm{H} 4 \mathrm{~A} & 109.5 & \mathrm{H} 7 \mathrm{~A}-\mathrm{C} 7-\mathrm{H} 7 \mathrm{~B} & \\ & & & 0.7(4) \\ \mathrm{N} 2-\mathrm{C} 2-\mathrm{C} 3-\mathrm{N} 1 & -0.7(3) & \mathrm{C} 1-\mathrm{N} 2-\mathrm{C} 2-\mathrm{C} 3 & 0.5(3) \\ \mathrm{N} 2-\mathrm{C} 1-\mathrm{S} 1-\mathrm{C} 5 & 104.2(3) & \mathrm{C} 1-\mathrm{N} 1-\mathrm{C} 3-\mathrm{C} 2 & -61.4(3) \\ \mathrm{N} 1-\mathrm{C} 1-\mathrm{S} 1-\mathrm{C} 5 & -77.0(3) & \mathrm{C} 1-\mathrm{S} 1-\mathrm{C} 5-\mathrm{C} 6 & -177.7(3) \\ \mathrm{C} 2-\mathrm{N} 2-\mathrm{C} 1-\mathrm{N} 1 & -0.4(3) & \mathrm{C} 4-\mathrm{N} 1-\mathrm{C} 3-\mathrm{C} 2 & 178.1(3) \\ \mathrm{C} 2-\mathrm{N} 2-\mathrm{C} 1-\mathrm{S} 1 & 178.7(2) & \mathrm{C} 4-\mathrm{N} 1-\mathrm{C} 1-\mathrm{N} 2 & -0.9(4) \\ \mathrm{C} 3-\mathrm{N} 1-\mathrm{C} 1-\mathrm{N} 2 & -0.1(3) & \mathrm{C} 4-\mathrm{N} 1-\mathrm{C} 1-\mathrm{S} 1 & 121.8(3) \\ \mathrm{C} 3-\mathrm{N} 1-\mathrm{C} 1-\mathrm{S} 1 & -179.1(2) & \mathrm{C} 7-\mathrm{C} 6-\mathrm{C} 5-\mathrm{S} 1 & \end{array}$

Hydrogen-bond geometry $\left(\AA,{ }^{\circ}\right)$

\begin{tabular}{lllll}
\hline$D-\mathrm{H} \cdots A$ & $D-\mathrm{H}$ & $\mathrm{H} \cdots A$ & $D \cdots A$ & $D-\mathrm{H} \cdots A$ \\
\hline $\mathrm{N} 2-\mathrm{H} 2 \cdots \mathrm{Br} 1^{\mathrm{i}}$ & $0.84(3)$ & $2.46(3)$ & $3.246(2)$ & $158(3)$ \\
$\mathrm{C} 2-\mathrm{H} 2 A \cdots \mathrm{Br} 1^{\mathrm{ii}}$ & 0.93 & 2.84 & $3.723(4)$ & 159 \\
$\mathrm{C} 3-\mathrm{H} 3 \cdots \mathrm{Br} 1^{\mathrm{iii}}$ & 0.93 & 2.91 & $3.757(3)$ & 152 \\
$\mathrm{C} 4-\mathrm{H} 4 B \cdots \mathrm{Br} 1$ & 0.96 & 2.87 & $3.737(3)$ & 151 \\
$\mathrm{C} 5-\mathrm{H} 5 B \cdots \mathrm{Br} 1^{\text {iv }}$ & 0.97 & 2.89 & $3.814(3)$ & 161
\end{tabular}

Symmetry codes: (i) $x,-y+1 / 2, z-1 / 2$; (ii) $-x+1, y-1 / 2,-z+1 / 2$; (iii) $-x+1,-y+1,-z+1$; (iv) $-x+2,-y+1,-z+1$. 